\title{
Recent Developments in Human Odor Detection Technologies
}

\section{Sichu $\mathrm{Li}^{*}$}

MITRE Corporation, 7515 Colshire Drive, McLean, VA 22102, USA

${ }^{*}$ Corresponding author: Sichu Li, MITRE Corporation, 7515 Colshire Drive, McLean, VA 22102, USA, E-mail: sichu@mitre.org

Citation: Sichu Li (2014) Recent Developments in Human Odor Detection Technologies. J Forensic Sci Criminol 1: S104. doi: 10.15744/2348-9804.1.S104

Received Date: March 05, 2014 Accepted Date: June 09, 2014 Published Date: June 11, 2014

\begin{abstract}
Human odor detection technologies have drawn attention due to the wide possibility of potential applications they open up in areas such as biometrics, criminal investigation and forensics, search for survivors under rubble, and security checkpoint screening. Gas chromatography/mass spectrometry (GC/MS) has been the most successful and powerful analytical approach developed to date for human odor analysis, and hundreds of human odorants have been identified using this tool. GC/MS has already enabled a good understanding to be obtained regarding human odor composition. Over the past two decades, research and development of E-nose technologies has accelerated at a fast pace, and in time may provide a complementary technology to those based on GC/MS. During the past several years, proof of concept has been demonstrated on the application of E-noses for real-time human odor detection and classification. In this review, the current state of technologies will be given on human odor analysis, detection, and classification, along with a discussion of each technology with a specific focus on recent developments. Technologies covered in this article include: various E-nose technologies; as well as gas chromatography integrated with mass spectrometry, ion mobility spectrometry, or other gas detectors. Other technologies will also be described such as optical sensors that have recently emerged for human odor detection, and the possibilities of exploiting absorbance spectroscopy and hyperspectral imaging.
\end{abstract}

Keywords: Human odor; Human odorant; Human odor detection technology; GC/MS; GC/IMS; E-nose

Abbreviations: API-Atmosphere Pressure Interface; CCD: Charge-coupled Device; CNT: Carbon Nanotubes; DNA: Deoxyribonucleic Acid; FID: Flame Ionization Detection; FTIR: Fourier Transform Infrared Spectroscopy; GC: Gas Chromatography; HR: High Resolution; HS: Head Space; HSI: Hyperspectral Imaging; IMS: Ion Mobility Spectrometry; LOD: Limit of Detection; MCC: Multi Capillery Column; MS: Mass Spectrometry; OP-FTIR: Open-path Fourier Transform Infrared Spectroscopy; PCA: Principal Component Analysis; PDID: Pulsed-discharge Ionization Detector; ppb: parts per billion; ppm: parts per million; ppt: parts per trillion QC: Quartz Crystal; QCM: Quartz Crystal Microbalance; SAW: Surface Acoustic Wave; SPME: Solid Phase MicroExtraction; TD: Thermal Desorption; VOC: Volatile Organic Compound

\section{Introduction}

Detection of human odor has become a subject of significant research interest because of the rich information on human body chemistry that can be extracted from human odor and the wide variety of potential applications this opens up. Since each person's body produces unique odors regardless of diet, human odor can be used as a biometric for non-invasive and non-collaborative subject identification [1-3]. The tracking of subject location as well as the path or objects in which the subject comes into contact via human odor detection is also a potential application for criminal investigation and forensics [4,5]. The chemical profile of human odor is affected by a person's stress, which could be used for detecting deception such as in security checkpoint screening [6]. In addition, the chemical components in a person's odor are determined by their health condition and can be exploited for disease diagnoses [7-9]. Furthermore, human odor detection could have a potential application in assisting rescue workers in searching for survivors under rubble after disasters such as an earthquake or building collapse [10-12].

Humans have a distinctive odor signature as evidenced by the ability of canines to track missing people and find them buried under rubble. This odor signature is unique to each person as evidenced by studies that show canines can positively match the scents of individuals down to identical and non-identical twins [13]. This is likely due to their ability to detect odor concentrations in the range of ppb-ppt, as has been shown by experiments in their detection of organic and inorganic compounds [14], and suggests the limit of detection needed to uniquely identify human odor signatures. Although canines can be trained for human odor detection, there are shortcomings to using this approach such as cost (e.g. \$50,000 per dog per year), limited working hours, and their 6-8 year working lifetime [15]. Moreover, the number of trained sniffer dogs in the world is limited. This motivates the need to develop odor detection instruments that can operate 24 hours a day as needed, and have the ability to provide unambiguous, reproducible, and reliable detection and identification of human odor at the level of the canine nose. Although there is currently no instrument that can substitute for the sensitivity and specificity of a well- trained canine nose, substantial progress has been made towards achieving this goal via a variety of approaches. 
The most powerful analytical approach is GC/MS, and it has been successfully used for detecting volatile organic compounds (VOCs) in human odor [16-21]. This method provides the lowest detection limit for VOCs, which can achieve the ppt level when using a preconcentrating process [22]. However, it is typically not possible to perform the analysis in real time which is needed for rapid detection in many real-world applications. This has driven development of real-time (on-line) or near real-time detection technologies for human odor. Progress has been made in the past decade in developing sensor systems that show promise for creating simple and rapid on-line systems. Near real-time detection systems have been developed and applied to human odor detection, including ion mobility spectrometry (IMS) coupled with GC (GC/IMS) or multicapillery columns (MCC/IMS) [12,23], as well as GC integrated with gas sensors [24-27]. Real-time sensor systems, such as E-noses [28-31], mass spectrometers [1], optical sensors [32], etc., have demonstrated the capability to detect human odor. However, the detection sensitivity and specificity of these systems still need improvements to reach a level that is comparable to the ability of a mammalian nose for detecting human odor.

This article will first briefly summarize human odor and its perception by olfactory systems, and then give an overview of the current human odor detection technologies. This will be followed by a discussion of individual technologies with a focus on recent developments for human odor detection and classification.

\section{Human odor and its perception}

An odorant is a substance capable of eliciting an olfactory response whereas odor is the sensation resulting from stimulation of the olfactory [33]. Human odor is caused by a complex array of volatile chemicals. They are emitted from different parts of the human body that are prone to odor production (e.g., axillae, scalp, hands and feet, groin, and oral cavity) [9,34]. Based on where they are emitted from, human odor is usually divided into three common categories: 1) skin odor; 2) the odor released from the oral cavity (e.g., breath odor); and 3) the odor emitted from human excreta (e.g., urine) [9]. Human odors are also categorized into three groups depending on their nature: 1) Primary odor, in which the production of odorants is genetically controlled and thus is stable over time regardless of diet and environmental factors; 2) Secondary odor, in which the odorants present are due to diet and environmental factors; and 3) Tertiary odor, which is composed of odorants from outside sources, including cosmetic and skin care products (e.g., soaps and perfumes) [5].

The major constituents of human odor are VOCs. Most of the relevant scientific research pertaining to chemical components in human odor has been focused on axillary (armpit) and planter (foot) sweat [9,16-18,5,35-39]. It has been demonstrated that human skin odor consists of $\mathrm{C}_{6}$ to $\mathrm{C}_{11}$ straight chain, branched and unsaturated acids [5,9], alcohols, ketones, aldehydes [34], carbonyls, and some steroids [5]. Essential contributors to skin odor were identified as terminally unsaturated acids, 2-methyl $\mathrm{C}_{6}$ $\mathrm{C}_{10}$ acids, and 4-ethyl $\mathrm{C}_{5}-\mathrm{C}_{11}$ acids, along with (E)-3- methyl-2-hexenoic acid [9,23,27,38]. For disease diagnosis and monitoring purposes, the analysis of the composition of breath has drawn increased interest in recent years. It was discovered that, although the VOCs in breath vary greatly from person to person, they are found to commonly include isoprene, ethane, pentane, methanol and acetone [40,41]. Most of the analyses of VOCs in human urine have been conducted for the purposes of disease diagnosis due to it containing volatile metabolites. Common VOCs of normal urine are identified as 2- butanone, 2-pentanone, 4-heptanone, dimethyl disulfide, alkyl furane, pyrrole, and carvone [42]. However, relatively little effort has yet been devoted to characterize urine odor either as a component in human body odor or as a marker for disease diagnostics [9].

Clearly, human odor is formed from a mixture of different odorants. An olfactory's perception of different components in a mixture of odorants is very different from how they would be perceived independently. This is because of interactions that occur between the different odorants in the mixture. An odorant can act as an additive agent, a counteractant, or a masking agent to the other odorants [33]. Therefore, a mixture of two odorants can generate an odor that is equal to or less than that of either one of the odorants, or produce an odor that is equal to, greater than, or less than the sum of the two odorants. Furthermore, the ratio of the two odorants in the mixture can also affect the odor property. There are hundreds of odorants in human odor and their complex interactions are still not well understood. However, it may be just these complex interactions that cause many odors to have a unique property that allows them to be distinguished by olfactory systems.

\section{Overview of human odor detection technologies}

Depending on the target application, human odor detection relies on the detection of different categories or components of odor. For instance, the primary odor can be used for subject identification while the secondary odor can be exploited for disease diagnosis and deception identification. Detection for subject identification is based on the chemical signatures of human odor, which is determined by the components of the odor and their differential information. Disease diagnosis depends on the presence of one or a few odorants, e.g. acetone and other ketones for diabetes mellitus [43]. This variation in detection purposes may be met by different detection technologies. Table 1 lists a variety of technologies that have been applied to detecting human odor.

The GC/detector technologies detect individual VOC components of an odor sample by first separating the complex VOC mixture (odor sample) into individual VOCs and then detecting them sequentially using the detector. The detector can be a MS system, IMS detector or a gas sensor. These technologies are suitable for discovering what VOC components are present in an odor sample. They can be also employed for detecting known VOC targets in human odor samples for various applications, as well as for human 
odor analysis for a better understanding of its content. Typically, sample collection and preconcentrations are needed in order to increase the probability of detection. This step, together with GC separation, provides these technologies with a sensitive detection capability, with limits of detection (LOD) as low as the ppb to ppt level [22]. However, the tradeoff is longer detection times that preclude real time application.

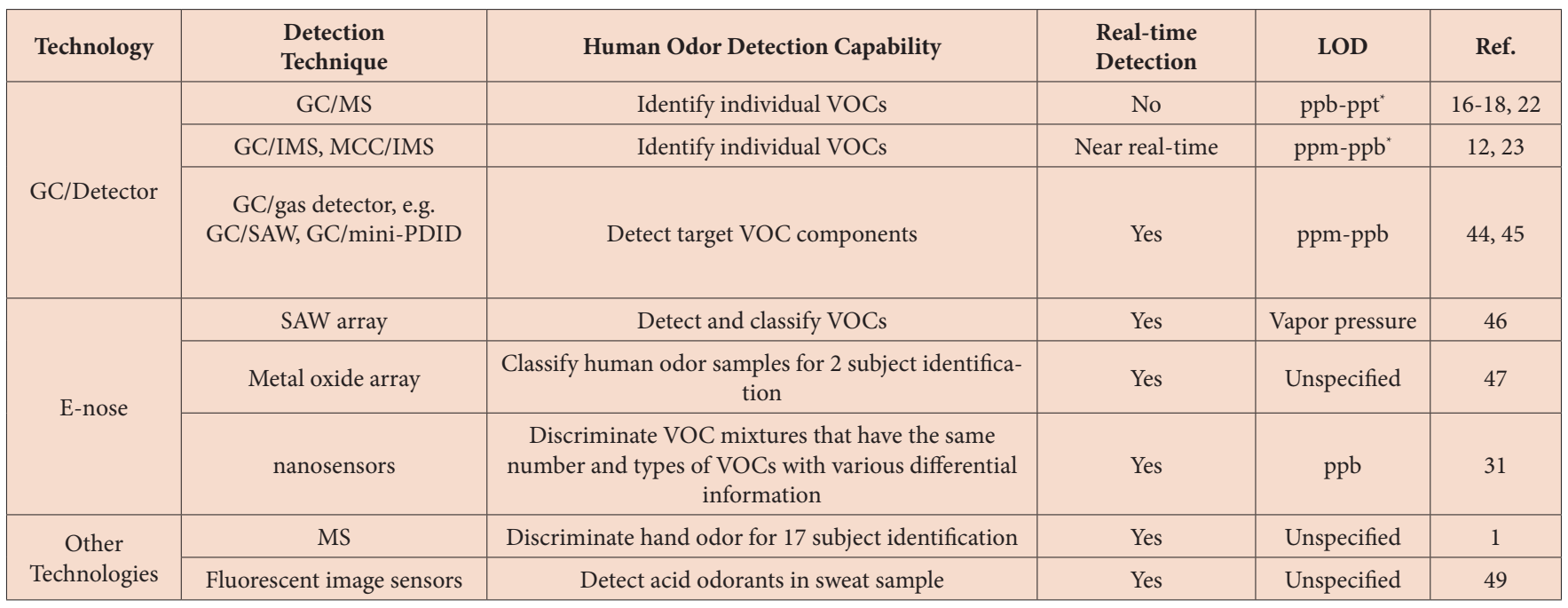

"With a preconcentration process

Table 1: Summary of Technologies Applied for Human Odor Detection

E-noses utilize an array of gas sensors to give a fingerprint response to a given analyte. They were originally designed to mimic an olfactory to perceive an odor. Unlike the GC/MS systems, E-noses detect odor in real time. They are composed of a sensor array to not only detect but also discriminate among complex odors [50-53]. The sensor array typically consists of a group of non-specific sensors of which each differentially responds to a subset of VOCs. The detection and identification of a particular odor is based on a unique combined response pattern from all sensors rather than a response pattern from a particular sensor. In addition to the sensor array, the response pattern recognition algorithm is another key component of an E-nose system that determines how well the E-nose identifies odors [1]. Detection using E-noses does not require a preconcentration step, and the detection sensitivity is in the ppm to ppb range. There is very limited literature on the application of E-noses to human odor detection.

Although the GC/detector systems and E-noses are the commonly employed technologies for human odor detection and classification, other technologies such as optical sensors are complementary approaches that could be used for certain applications, e.g. standoff subject detection.

\section{GC/Detector systems for human odor detection}

Traditional analytical methods for detecting VOCs in human odor use a combination of GC with a universal detection technique such as flame ionization detection (GC/FID) [24], mass spectrometry (GC/MS) $[8,16,17,33$ ], or ion mobility spectrometry (GC/IMS) [12]. In such systems, a preconcentration process is typically carried out. Headspace solid phase microextraction (HS-SPME) is a well-established and commonly used preconcentration technique that is based on partitioning and achieving an equilibrium of analytes between different phase matrices. When sorbent-based sampling is employed for collecting VOCs in human odor, desorption of analytes for analysis is achieved with the aid of a solvent or by using thermal desorption (TD) [9]. By using an appropriate preconcentration technique, the detection sensitivity can be further improved to the ppt level [22]. GC also can be integrated with gas sensors to enhance the detection capability, because the GC separation can effectively reduce possible interference for target analyte detection [24-27].

\section{GC/MS}

Table 2 summarizes some of the VOCs that have been detected in human odor using the GC/MS method. Mass spectrometry offers the capability to detect a broad spectrum of VOC components in human odor, and thus has been widely used for human odor identification. Bernier et al. [18,38] reported that GC/MS with TD allowed the identification of more than three hundred VOC components in human skin odor, including alcohols, aldehydes, acids, amides, amines, aromatics, esters, and ketones. GC/MS with HS-SPME also has been employed to identify several dozens of VOCs in human male sweat samples [16,21]. Furthermore, it was recently demonstrated that multi-dimensional GC/MS (GC-GC/MS) could effectively identify VOCs in complex samples, e.g. VOCs in human urine [54]. The multidimensional GC-GC separation allows a more efficient separation and increases the peak capacity. Sandgruber et al. [17] used a two-dimensional high resolution GC/MS (HR-GC/MS) to identify thirteen VOCs in human milk with detection concentrations in the range of $10^{-1}-10^{1} \mu \mathrm{g} / \mathrm{kg}$, which is equivalent to $10^{-1}-10^{1} \mathrm{ppb}$. 
The GC/MS method typically employs batch detection that involves multiple separate analytical steps: VOC sampling, sample transportation, analyte preconcentration, separation via chromatography, detection, data transmission, and post-data analysis. Although the method is very useful for VOC trace detection, it is quite time-consuming and requires users be well trained before they can utilize the instrument for VOC analysis. In addition, VOC sample loss could occur during sample transportation and thus may affect the concentration measurement. Since the sample loss rate varies for different VOCs, any results that are related to a differential concentration among different VOC components in an odor sample could be affected by sample transportation.

\begin{tabular}{|c|c|c|}
\hline Human Odor Categories & Detected VOCs & Ref. \\
\hline Human skin & $\begin{array}{l}\text { Lactic acid, aliphaticfatty acids, butanal, 3-methyl-butanal, 2-methyl-butanal, pentanal, hexanal, heptanal, octa- } \\
\text { nal, phenylacetaldehyde, nonanal, decanal, and undecanal }\end{array}$ & 9,18 \\
\hline Human body & 2-nonenal, hydrocarbons, alcohols, acids, ketones, and aldehydes & 9,19 \\
\hline Foot malodor & Acetic acid, butyric acid, isobutyric acid, isovaleric acid, propionic acid, valeric acid, and isocaproic acid & 9,20 \\
\hline Human male axillae & C6 - C11 normal, branched, and unsaturated acids & 9,21 \\
\hline Human male armpit sweat & $\begin{array}{l}\text { 2-Methyl-butanal, acetic acid, 3-methyl-butanal, 2-pentanone, 2-methyl-pentenal, lactic acid, isovaleric acid, } \\
\text { m-xylene, } \alpha \text {-pinene, 1-octen-3-ol, 6-methyl-5-hepten-2-one, m-cymene, benzenemethanol, nonan-2-one, } \\
\text { isobornyl acetate, p-cymen-7-ol, } \alpha \text {-bergamotene, geranyl acetone, } 2 \text {-pentadecanone, } \alpha \text {-selinene, dodecanoic } \\
\text { acid, tridecan-1-ol, benzophenone, tetradecanoic acid, 9-hexadecanoic acid methyl ester, isopropyl myristate, } \\
\text { isopropyl palmitate, squalene }\end{array}$ & 16 \\
\hline Breast milk & $\begin{array}{l}\text { Hexanal, octanal, nonanal, decanal, }(\mathrm{Z}) \text {-hex-3-enal, (E)-non-2-enal, (E)-dec-2-enal, (E,E)-deca-2,4-dienal, } \gamma \text { - } \\
\text { nonalactone, } \delta \text {-decalactone, } \gamma \text {-dodecalactone, oct-1-en-3-one, }(Z) \text {-octa-1,5-dien-3-one }\end{array}$ & 17 \\
\hline
\end{tabular}

Table 2: Human Odor VOCs Detected Using GC/MS.

\section{GC/IMS and MCC/IMS}

The ion mobility spectrometry (IMS) technique was developed in the early 1970s for detecting explosives and chemical warfare agents. Due to the capability to execute quick analysis of VOCs, IMS has been applied during the last decade for environmental monitoring [55,56], disease diagnosis [57-60,54,61,62], and human odor analysis [12,63]. IMS is based on the ionization of VOCs according to atmosphere pressure chemical ionization processes and the separation of the ions based on their mobility in an electric field [61].

IMS, when coupled with a preconcentration step using a SPME, can provide a LOD as low as 2 ng for VOC detection (e.g. piperronal) [63]. IMS is often integrated with GC columns [64,65] or multicapillery columns (MCCs) [57] to provide more sensitive detection due to the enhanced separation. MCCs distinguish themselves by comparatively high flow rates and high sample capacities in comparison to single narrow columns [57]. Table 3 lists human odor VOCs that were detected using MCCIMS systems [12,23]. Ruzsanyi et al. [23] demonstrated the detection of more than twenty VOCs emitted from human skin, out of which eight compounds were identified. The detection and identification steps were conducted in near real time without using a preconcentration step, yielding a LOD in the range of $10^{-1}-1 \mathrm{ppb}$, which is comparable to that obtained with a SPME GC/MS method [23]. Rudnicka et al. [12] used MCC-IMS to detect and identify twenty-three ubiquitous VOCs in human urine, and found that fourteen of them exist in the urine headspace (HS). Huo et al. [65] exploited GC/IMS to detect human odor VOCs that can be used as markers to detect humans trapped under collapsed buildings.

\begin{tabular}{|c|c|c|}
\hline Human Odor Categories & Detected VOCs & LOD (ppb) MCC/IMS (GC/MS) \\
\hline \multirow{9}{*}{ Human skin [21] } & 3-Methyl-2-butenal & 0.6 \\
\hline & n-Butylacetate & $0.2(0.1)$ \\
\hline & Benzaldehyde & $0.3(0.2)$ \\
\hline & 6-Methylhept-5-en-2-one & $0.7(0.3)$ \\
\hline & Octanal & $0.6(0.3)$ \\
\hline & 2-Ethyl-hexanol & 1.0 \\
\hline & Nonanal & 0.5 \\
\hline & Decanal & 0.3 \\
\hline & Ammonia, ethanol & unspecified \\
\hline Human urine $[12]$ & $\begin{array}{l}\text { 2-Butanone, 2-methyl-1-propanal, 2-methyl-2-butenal, 2- } \\
\text { pentanone, 3-hexanone, 3-methyl-2-butanone, 3-methylfuran, 3- } \\
\text { penten-2-one, 4-heptanone, acetone, dimethyl disulfide, dimethyl } \\
\text { sulfide, ethyl acetate, furan, isoprene, methanethiol, methyl } \\
\text { acetate, octanal, pentanal, propanal, pyrrole, toluene }\end{array}$ & unspecified \\
\hline
\end{tabular}

Table 3: Human Odor VOCs Detected Using MCC/IMS. 
Compared to MS, IMS typically provides less spectral resolution, and thus the compound identification capability is weaker. Also, for compound identification using a library, the current IMS library is not as universal as MS. However, IMS has attractive advantages over MS including short detection time, direct odor sampling, portability, easy operation, continuous operation, and low power consumption. Such advantages make MCC/IMS a compelling alternative to GC/MS for applications that require field deployment.

\section{GC/Gas sensor systems}

A surface acoustic wave (SAW) sensor is a type of gas sensor that has been combined with GC (GC/SAW) and explored for its capabilities in human odor detection. Fast GC/SAW, also called zNose, is a technique that integrates fast gas chromatography with surface acoustic wave sensing to provide a real-time, simple, low-cost detection [24-27]. A redeeming characteristic of the fast GC/ SAW method is that it does not require VOC extraction [66]. As shown in Figure 1(a), the headspace is sampled by pumping the gas through a valve to a trap where the VOCs are adsorbed. To detect the VOCs, as shown in Figure 1(b), the valve is switched to an injection process that causes helium gas to flow backwards through the trap and onto the GC column and then the SAW detector. The method permits a high reproducibility of detection and good sensitivity at the high pictogram to nanogram level, making it possible to detect sensitive living materials quantitatively [67]. Oh [66] employed the fast GC/SAW technique to detect VOCs of the Lavandula species, and analyzed the data using principal component analysis (PCA) to obtain the VOC composition profile data for the species. Table 4 provides a list of VOCs detected using a GC/SAW system and their detection LODs in air and in the headspace of water, which fall in the range of ppb [68]. GC/SAW also was used for analyzing human hand odor samples, from which significant differences were found between different subjects [44].
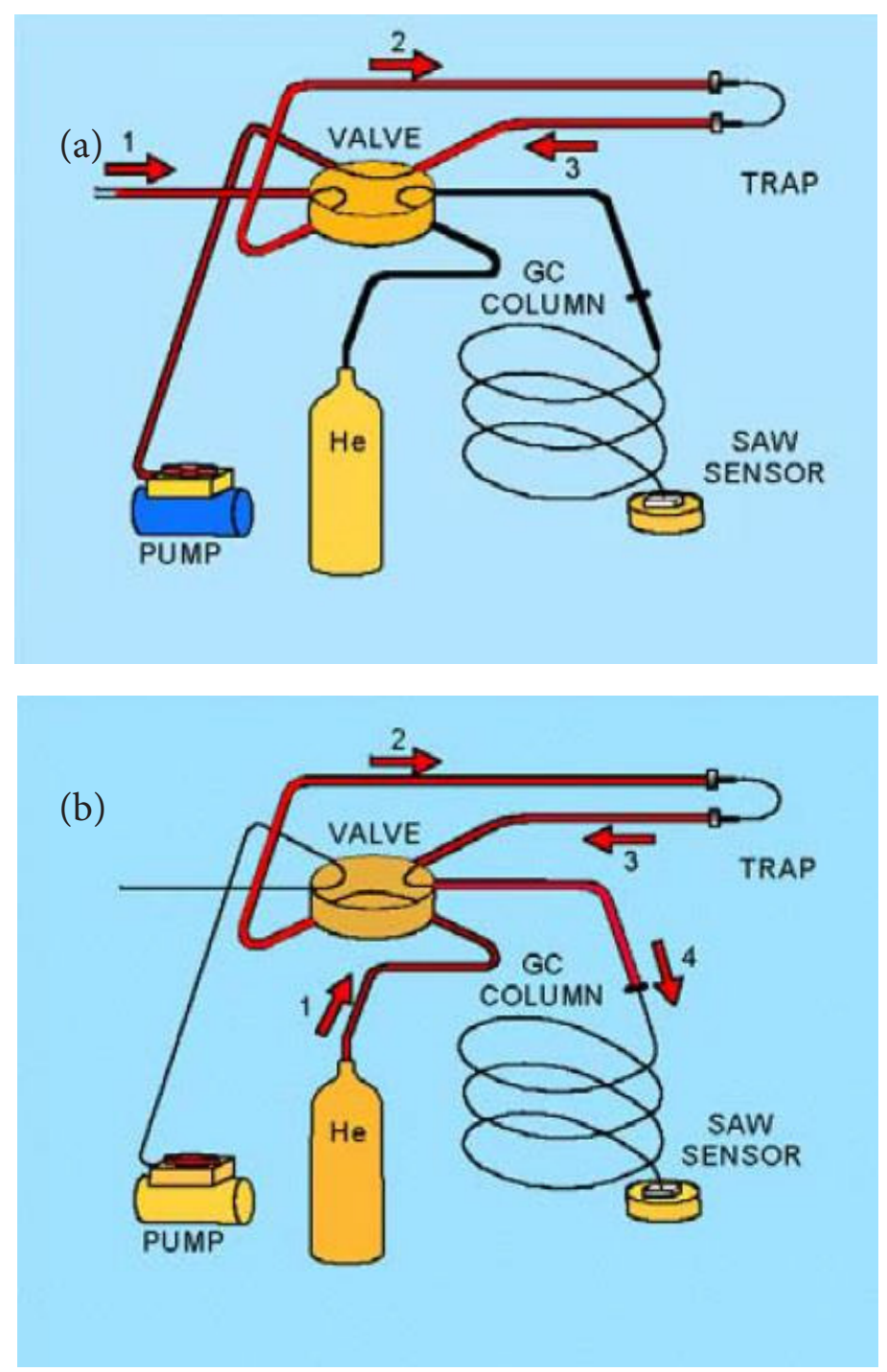

Figure 1 [68]: Schematic diagram of GC/SAW system: (a) Sampling and (b) Detection 
Another gas sensor system that can be integrated with a GC column is a miniature pulsed- discharge ionization detector (miniPDID) developed by Manginell et al. that allows identification of vapors by "sniffing" the VOCs [69]. The device is about 1 inch by 1 inch by 2 inches, can be coupled with commercially produced micro-GCs, and can run for nine hours on a single charge of helium. The mini-PDID can be used for detecting VOCs as bacterium biomarkers [45]. Since hexenoic acid is a unique VOC component in human sweat (not present in other animals) it can be used as a biomarker to detect the presence of humans. It was proved that the mini-PDID could find hexenoic acid at the ppb level and therefore could be employed as a detector for hidden human cargo [69], which has applications for human smuggling across national borders. As described by the developer, the PDID would integrate well with the SAW and micro-GCs to provide both sensitivity and selectivity: the ability to detect a few molecules of a given compound, and the ability to distinguish compounds from one another, respectively [69].

\begin{tabular}{|c|c|c|}
\hline VOC & $\begin{array}{c}\text { LOD }(\mathbf{p p b}) \\
\text { In Air }\end{array}$ & $\begin{array}{c}\text { LOD }(\mathbf{p p b}) \\
\text { Water HS }\end{array}$ \\
\hline Chloroform & 45 & 0.65 \\
\hline Cis-1,2-dichloroethene & 47 & 1.7 \\
\hline Benzene & 42 & 0.96 \\
\hline Carbon tetrachloride & 130 & 16.49 \\
\hline Trichloroethylene & 6.3 & 0.40 \\
\hline Toluene & 11 & 0.15 \\
\hline Tetrachloroethylene & 5.7 & 0.57 \\
\hline Ethylbenzene & 2.7 & 0.07 \\
\hline O-xylene & 2.5 & 0.11 \\
\hline $\begin{array}{c}1,1,2,2- \\
\text { tetrachloroethane }\end{array}$ & 3.6 & 0.56 \\
\hline
\end{tabular}

Table 4 [68]: List of VOCs Detected Using Fast GC/SAW and Their LODs.

The GC/gas sensor systems have advantages such as small dimensions, easy deployment in field operations, and low cost. In the past decade, efforts have been devoted to improving the gas sensor detection sensitivity such that the LODs now fall in the ppb level. However, these sensors require training prior to being applied for detecting target VOCs. Their identification capability is still not comparable to that of the GC/MS method, and their robustness could be an issue due to degraded performance from the effects of environmental operating conditions such as humidity, temperature, etc.

\section{E-noses}

E-noses are sensor systems that are designed to mimic the mammalian nose. They are typically composed of an array of non-specific sensors that respond to either individual or classes of volatile chemicals. As shown in Figure 2, the odorant's molecules are drawn into the E-nose using sampling techniques, e g. headspace sampling, diffusion methods, bubbling, or pre- concentrating. When the odorants land on the sensor array and interact with the sensing materials, a reversible change in a chemical or physical property is induced. Such change is transduced into an electrical signal that is recorded and processed. Pattern recognition techniques are applied to analyze the sensor data to detect, classify, or identify the analytes. Different sensing techniques have been utilized for constructing E-noses, which include SAW [30,46,70-72], quartz crystal microbalance (QCM) [73-76], and chemiresistors based on metal oxides [29,47,67,77-79], conducting polymers [32,80,81], carbon nanotubes (CNTs) $[33,48,82]$, or a combination of multiple techniques [83].

\section{Sensing techniques for E-noses}

The SAW sensor transducer is mass sensitive. The sensor is composed of a substrate of quartz that is cut at a crystalline angle to support a surface wave, as well as a chemically sensitive thin film that is coated on the quartz surface. Since the quartz is a piezoelectric material, it converts surface acoustic waves to electric signals. This occurs when the chemically sensitive thin film adsorbs specific molecules, causing the mass of the film to increase, thereby causing the acoustic waves to travel more slowly. This change can be detected by the sensor microelectronics once the acoustic wave is converted to an electric signal [72].

QCM is another type of microbalance mass sensor similar to the SAW sensor. The major difference between SAW and QCM is that the former employs a surface acoustic wave sensor while the latter uses a bulk acoustic wave sensor. Its sensing mechanism is based on the shift in the quartz crystal (QC) resonant frequency due to the adsorption of gas molecules onto the sensing films [73-76]. 


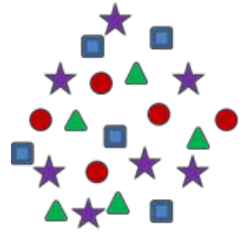

Odorants

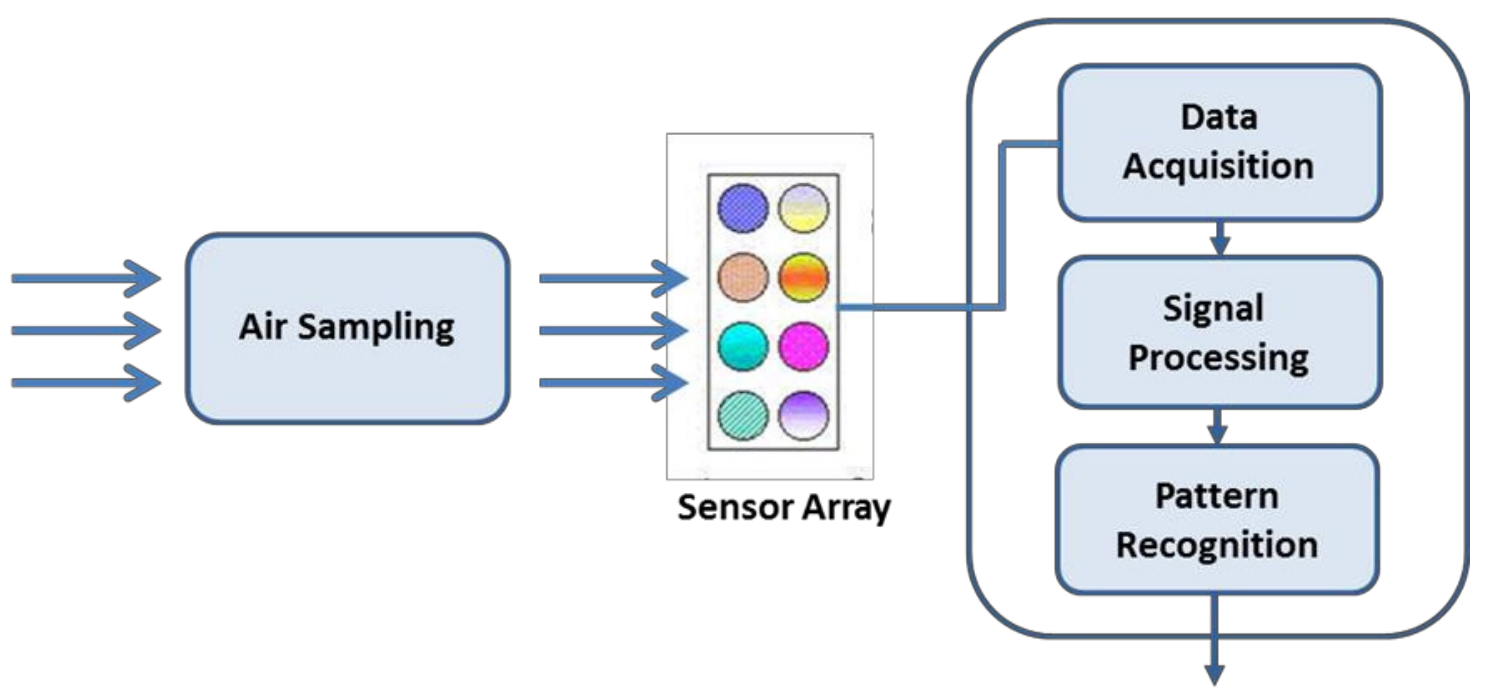

Result

Figure 2: Concept of E-Nose System Operation Principle.

Chemiresistors are sensors that translate a change in the concentration of chemical vapors into electrical signals. The conducting materials include metal oxide semiconductors, conducting polymers, or CNTs. When analytes collect upon the surface of these materials certain reactions occur resulting in electron transfer from the analytes to the conducting materials, which trigger changes in the electrical signals [29,31,32]. An example of a CNT transistor is schematically demonstrated in Figure 3 [82].

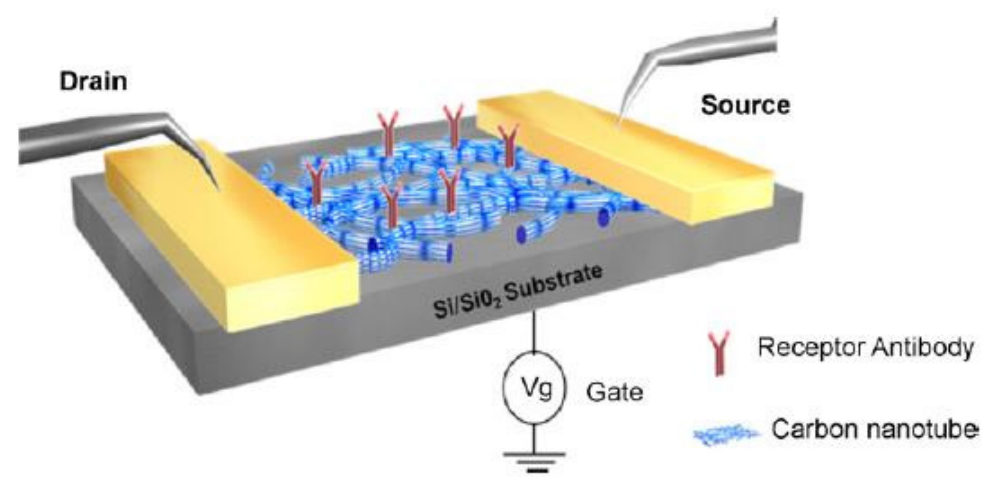

Figure 3 [82]: Schematic diagram of a CNT transistor device.

Apart from the sensing mechanism, much effort in current E-nose research has focused on functional materials for sensing applications $[46,82]$. Nanomaterials have high surface area to volume ratios and possess unique chemical and physical properties, and therefore are chosen as sensing materials. CNT-based sensors have drawn much attraction due to inherent CNT properties such as extremely small size, high strength, high electrical and thermal conductivity, and high specific surface area [82]. An E-nose based on an array of DNA-coated nanosensors has been demonstrated for odor detection [31,48]. The use of DNA oligomers as odorant receptors could improve the detection specificity, and using the small-size CNTs as the sensing transducer would allow for the construction of a sensor array with a huge number of sensors. Such a system represents a promising step forward towards developing a new generation of devices for electronic olfaction, that would be capable of matching or exceeding the demonstrated sensitivity and selectivity of trained biological olfactory systems [48].

\section{E-noses for human odor detection and classification}

E-noses have been used for various applications such as monitoring food and beverage quality, environmental monitoring, and biomedicine. Although there are very few reports regarding the application of E-noses for human odor detection and classification, progress has been made during the past several years in improving the E-nose capability for human odorant detection and classification [46,48], and even in human odor classification [31,32]. The latter in particular requires the E-nose to be capable of differentiating VOC components in complex VOC mixtures. Demonstration of this capability, which mimics how odor is perceived by a mammalian nose, should be considered a remarkable step forward in developing an E-nose that truly functions as an artificial nose. 
Jha et al. [46] used an E-nose based on three SAW sensors to detect fourteen human odorants with concentrations equivalent to their vapor pressures. The resultant sensor data was analyzed using PCA, and it was found that the odorants were separated into four families: aldehydes, acids, ketones, and alcohols. Johnson et al. [48] fabricated an array of nanosensors based on single-wall CNTs coated with various DNA oligomers with different sequences, which act as receptors to different human odorants. It was demonstrated that single DNA oligomer sequences can provide differential and robust responses to homologous series of aldehydes (octanal, nonanal, and decanal) and carboxylic acids (propionic acid, hexanoic acid, and octanoic acid) found in human breath.

Remarkable work has been conducted to demonstrate the capability of E-noses for human odor detection and classification [31,47]. Kybert et al. [31] fabricated DNA coated CNT nanosensors and tested their responses to individual VOCs found in human odor, as well as complex mixtures formed with 17 human non-axillary skin odorants that more closely resemble "real-world" odor samples. It was discovered these nanosensors are able to not only detect individual VOCs at the ppb level within seconds, but also to discriminate between analytes with similar structures, e. g. three isomers of pinene. When testing the sensor capability to discriminate between VOC mixtures, 17 VOCs (most of them are human non-axillary skin odorants) are used to form a mixture (parent mixture), from which several other "spike" mixtures are created that have one of the VOCs at a higher concentration than that in the parent mixture (i.e., $2 \mathrm{x}, 5 \mathrm{x}$, and 10x). It was demonstrated that the nanosensors are able to discriminate the headspace samples of "spike" samples against the parent sample. Wongchoosak et al. [47] used a metal oxide based E-nose to detect and classify human odor samples collected from two subjects who have similar lifestyle and activities. It was also reported that deodorant does not affect the subject identification. Although the number of subjects used in this test is very limited and the method requires further validation, the preliminary results suggest that this E-nose could be applied for human body odor biometrics.

\section{Other technologies}

\section{Mass spectrometry}

A MS-based system, which was developed by Sociedad Europra De Análisis Diferecial de Movilidad SL (SEADM), was recently used to perform real-time human subject identification based on the classification of the subjects' hand odors [1]. The system comprises: 1) a vapor collector that drives the skin odor emanation from a hand to the ionizer; 2) an ionizer where the vapors contact with the ion cloud; and 3) an atmosphere pressure interface (API) MS. The system collects a mass spectrum every 0.1 second, and a set of mass spectra collected within the time period is used to form a data matrix for analysis. It was reported that, when using a machine learning classification model constructed using hand odor samples from 13 subjects, an $85 \%$ success rate was achieved in matching other batches of hand odor samples collected from the same group of people [1]. The subject identification was performed in real time. Although the success rate demonstrated in this work is relatively low for a biometric application, and the number of subjects tested is not high enough for strong statistical significance, the work provides evidence that a human subject has a distinctive odor signature.

\section{Optical sensing}

Liu et al. [49] recently reported their work on developing a fluorescent image system for human sweat odor detection. The sensing substrate in the system is an agarose-gel fluorescent film that contains a $\mathrm{pH}$ sensitive fluorophore, quinine sulfate. A change in fluorescence occurs when volatile organic acids in human sweat collect on the sensing substrate, which can be detected by using a high-resolution charge-coupled device (CCD) camera. Development of different sensors to detect other VOC components such as aldehydes and ketone is underway that may eventually allow the construction of a multiplex detection system for simultaneous detecting odorants with different types of chemical structures.

\section{Absorbance spectroscopies possibly for human odor detection}

Absorbance spectroscopies are another potential approach for detecting VOCs in human odor, especially for non-collaborative standoff detection. VOCs strongly absorb energy at mid- to far- infrared (IR; ca. 3-1,000 $\mu \mathrm{m}$ ) wavelengths, and provide a means for detecting VOCs at low concentrations [84]. Fourier transform infrared spectroscopy (FTIR) has been widely used for analysis of many organic compounds, and open-path FTIR (OP-FTIR) is a direct extension of this method for open spaces ranging from meters to a kilometer [84]. It was reported that this method can detect VOCs at the ppt level [85]. Several portable laser absorption spectroscopic (LAS) techniques are also possible means for trace gas detection, and have been utilized for detecting VOCs (acetone, isoprene, methanol, etc.) at concentration levels of ppm - ppt $[84,86]$.

Hyperspectral imaging (HSI) systems were originally developed for remote sensing imagery of the earth, but have since found applications in diverse fields such as food science, pharmaceuticals, medical diagnostics, and forensic trace analysis [87]. Hyperspectral imaging works by collecting images of a viewed scene within numerous limited spectral bands, and then combining these sets of images to form a three-dimensional hyperspectral data cube. The latter can then be processed and analyzed to enhance features in the imagery or extract specific information. Spectral resolution is the key discriminator in the precision of HSI systems, and the detection of large numbers of fairly narrow frequency bands by a HSI scanner makes possible the identification of objects because of the variation in reflectivity and thermal emission of materials within the image scene. Passive HSI systems can be optimized to select specific wavelengths of interest related to particular materials, and it is possible to tune the narrow bands to maximize the visibility of human sweat of a subject appearing in the imagery [34]. Although HSI methods have been explored for 
Journal of Forensic Science \& Criminology

various applications such as earth remote sensing, plume imaging, and explosive standoff detection, their capabilities for human odor detection are yet to be fully studied and understood. If these methods prove fruitful, they would be additional tools that could provide complementary application features to the current technologies.

\section{Conclusions}

Human odor carries rich information on human body chemistry and its detection has a wide variety of potential applications in areas such as biometrics and forensic investigation, disease diagnostics, survivor rescue, etc. Current approaches employed for human odor detection have one or both of the following capabilities: 1) Detect, discriminate between, and/or identify individual VOCs in human odor samples; 2) Detect and discriminate between odor samples (a mixture of VOCs) of different subjects. GC/ detector systems such as GC/MS, GC/IMS, and MCC/IMS have demonstrated high sensitivity for detecting individual VOCs.

GC/MS has been used as a powerful analytical tool to successfully identify hundreds of VOCs in human odor, allowing for a more in-depth understanding of its composition. GC/IMS and MCC/IMS systems provide real-time detection capability. Although its identification capability is not as strong as that of GC/MS, GC/IMS and MCC/IMS systems have advantages that include continuous operation, easy field deployment, and low cost.

E-noses have been developed for various applications of VOC detection and discrimination. However, their ability to detect and discriminate between different subject's odor samples, as well as different mixtures formed of the same number and types of human odorants, have only been preliminarily demonstrated recently. While more efforts need to be devoted to further validate these approaches, the preliminary results can be considered a remarkable step toward developing a truly artificial nose. During the last decade, a considerable amount of work has been done to improve the E-nose detection sensitivity down to the ppm to ppb level. However, the specificity of identification and the robustness still remains a huge challenge. Future efforts in developing a truly artificial nose must focus on implementing those natural nose functions that have not been implemented to date. This includes enhancing the VOC molecule recognition capability, developing and incorporating a molecule sorting mechanism that mimics the mucus action in the natural nose, stabilizing the operating conditions (temperature and humidity) to ensure the molecule recognition capability, as well as developing an appropriate air sampling mechanism to enhance VOC collection efficiency.

While GC/detector systems and E-noses currently are major technologies that have been explored for human odor detection, other technologies such as absorption spectroscopy may be worth exploring due to the strong adsorption features of VOCs in the mid- and far-IR spectral regions. HSI systems have been employed for standoff chemical detection, and it is conceivable that such systems could be adapted for standoff human odor detection, thereby providing an additional complementary tool to current capabilities in human odor detection.

Considerable progress has been made during the past decade in developing instruments that have the sensitivity and specificity to detect and discriminate human odor. With continued research and the innovative integration of new technologies, there is the reasonable expectation that within the next decade the sensitivity and specificity will increase to the point that these instruments have human odor detection capabilities equivalent to a canine nose. For example, with the nanofabrication techniques and development of nanomaterials that is currently rapidly emerging, it is conceivable that E-noses could be built with hundreds of nanosensors that incorporate bioreceptors for recognizing human odorants. The emerging technology in human odor detection is at the verge where, within our lifetime, artificial noses will become common devices that are used to improve our daily lives as essential aids in food spoilage, disease detection, detection of explosives, search for survivors under rubble, security at airports, and many more unforeseen applications.

\section{Acknowledgments}

The author would like to thank MITRE Corporation for the financial support for this work. Appreciation is also given to Dr. Carl Picconatto for reviewing this manuscript and his constructive comments.

\section{References}

1. Rodriguez-Lujan I, Bailador G, Sanchez-Avila C, Herrero A, Vidal-de-Miguel G (2013) Analysis of pattern recognition and dimensionality reduction techniques for odor biometrics. Knowledge-Based Systems 52: 279-89.

2. Gibbs MD (2010) Biometrics: body odor authentication perception and acceptance. ACM SIGCAS Computers and Society 40: 16-24.

3. Oyeleye CA, Fagbola TM, Babatunde RS, Adigun AA (2012) An exploratory study of odor biometrics modality for human recognition. Int J of Eng Res \& Tech 1: $1-9$.

4. Stockham RA, Slavin DL, Kitt W (2004) Survivability of human scent. Forensic Science Communications 6: 1-6.

5. Prada PA, Furton KG (2008) Human scent detection: A review of its developments and forensic applications. Revista de Ciencias Forenses 1: 81-7.

6. Rolfe B, Li S (2012) Human odor: Sniffing out identity and deception. The MITRE Corporation, McLean, Virginia.

7. Thorn RM, Greenman J (2012) Microbial volatile compounds in health and disease conditions. J Breath Res 6: 024001.

8. Buszewski B, Rudnicka J, Ligor T, Walczak M, Jezierski T, et al. (2012) Analytical and unconventional methods of cancer detection using odor. Trends in Analytical Chemistry 38: 1-2. 
9. Pandey SK, Kim K-H (2011) Human body-odor components and their determination. Trends in Analytical Chemistry 30: 784-96.

10. Frederickx C, Verheggen FJ, Haubruge E (2011) Biosensors in forensic sciences. Biotechnologie, Agronomie, Société et Environnement 15: 449-58. 11. Search and rescue dogs of the United States (SARDUS).

12. Rudnicka J, Mochalski P, Agapiou A, Statheropoulos M, Amann A, et al. (2010) Application of ion mobility spectrometry for the detection of human urine. Anal Bioanal Chem 398: 2031-8.

13. Pinc L, Bartoš L, Reslová A, Kotrba R (2011) Dogs discriminate identical twins. PLoS ONE 6: e20704.

14. Johnston JM (1999) Canine detection capabilities: Operational implications of recent R\&D finding. Institute for Biological Detection Systems, Auburn University.

15. Sivak M (2014) The dog's nose knows...or does it? Explosives detection by mechanical and electrical "noses". Illumin XV.

16. Mebazaa R, Rega B, Camel V (2011) Analysis of human male armpit sweat after fenugreek ingestion: Characterisation of odour active compounds by gas chromatography coupled to mass spectrometry and olfactometry. Food Chemistry 128: 227-35.

17. Sandgruber S, Much D, Amann-Gassner U, Hauner H, Buettner A (2012) Sensory and molecular characterisation of the protective effect of storage at -80 ${ }^{\circ} \mathrm{C}$ on the odour profiles of human milk. Food Chemistry 130: 236-42.

18. Bernier UR, Booth MM, Yost RA (1999) Analysis of human skin emanations by gas chromatography/mass spectrometry. 1. Thermal desorption of attractants for the yellow fever mosquito (Aedes aegypti) from handled glass beads. Anal Chem 71: 1-7.

19. Haze S, Gozu Y, Nakamura S, Kohno Y, Sawano K, et al. (2001) 2-Nonenal newly found in human body odor tends to increase with aging. J Invest Dermatol 116: $520-4$.

20. Caroprese A, Gabbanini S, Beltramini C, Lucchi E, Valgimigli L (2009) HS-SPME-GC-MS analysis of body odor to test the efficacy of foot deodorant formulations. Skin Res Technol 15: 503-10.

21. Zeng XN, Leyden JJ, Lawley HJ, Sawano K, Nohara I, et al. (1991) Analysis of characteristic odors from human male axillae. J Chem Ecol 17: 1469-92.

22. Hoffman EM, Curran AM, Dulgerian N, Stockham RA, Eckenrode BA (2009) Characterization of the volatile organic compounds present in the headspace of decomposing human remains. Forensic Sci Int 186: 6-13.

23. Ruzsanyi V, Mochalski P, Schmid A, Wiesenhofer H, Klieber M, et al. (2012) Ion mobility spectrometry for detection of skin volatiles. J Chromatogr B Analyt Technol Biomed Life Sci 911: 84-92.

24. Lammertyn J, Veraverbeke EA, lrudayaraj J (2004) zNose ${ }^{\mathrm{Tx}}$ technology for the classification of honey based on rapid aroma profiling. Sensors and Actuators B 98: 54-62.

25. Oh SY, Shin HD, Kim SJ, Hong J (2008) Rapid determination of floral aroma compounds of lilac blossom by fast gas chromatography combined with surface acoustic wave sensor. J Chromatogr A 1183: 170-8.

26. Oh SY, Ko JW, Jeong SY, Hong J (2008) Application and exploration of fast gas chromatography-surface acoustic wave sensor to the analysis of thymus species. J Chromatogr A 1205: 117-27.

27. Dissanayake D (2010) Acoustic waves. Sciyo publisher, Australia, Chapter 13: 299-314.

28. Lee DS, Jung JK, Lim JM, Huh JS, Lee DD (2001) Recognition of volatile organic compounds using SnO2 sensors array and pattern recognition analysis. Sensors and Actuators B 77: 228-36.

29. Rock F, Barsan N, Weimar U (2008) Electronic nose: current status and future trends. Chem Rev 108: 705-25.

30. Kybert NJ, Lerner MB, Yodh JS, Preti G, Johnson ATC (2013) Differentiation of complex vapor mixtures using versatile DNA-carbon nanotube chemical sensor arrays. ACS Nano 7: 2800-7.

31. Albert KJ, Lewis NS (2000) Cross reactive chemical sensor arrays. Chem Rev 100: 2595-2626.

32. Bornstein A, Damarla R, Lavery J, Morelli F, Schmeisser E (2010) Remote detection of covert tactical adversarial intent of individuals in asymmetric operations. U.S. Army Research Laboratory Special Report: 1-46.

33. Powers W (2004) The Science of Smell Part 3: Odor detection and measurement. Communication Services, Iowa State University: 1-4.

34. Wysocki CJ, Preti G (2004) Facts, fallacies, fears, and frustrations with human pheromones. Anat Rec A Discov Mol Cell Evol Biol 281: 1201-11.

35. Lenochova P, Roberts SC, Havlicek J (2009) Methods of human body odor sampling: the effect of freezing. Chem Senses 34: 127-38.

36. Zhang ZM, Cai JJ, Ruan GH, Li GK (2005) The study of fingerprint characteristics of the emanations from human arm skin using the original sampling system by SPME-GC/MS. J Chromatogr B Analyt Technol Biomed Life Sci 822: 244-52.

37. Akin J (2005) Characterization of human skin emanations by solid phase microextraction (SPME) extraction of volatiles and subsequent analysis by gas chromatography-mass spectrometry (GC-MS). Massachusetts Institute of Technology, USA.

38. Bernier UR, KlineDL, Barnard DR, Schreck CE, Yost RA (2000) Analysis of human skin emanations by gas chromatography/mass spectrometry. 2. Identification of volatile compounds that are candidate attractants for the yellow fever mosquito (aedes aegypti). Anal Chem 72: 747-56.

39. Fredrich E, Barzantny H, Brune I, Tauch A (2013) Daily battle against body odor: towards the activity of the axillary microbiota. Trends Microbiol 21: 305-12. 40. Miekisch W, Schubert JK, Noeldge-Schomburg GF (2004) Diagnostic potential of breath analysis--focus on volatile organic compounds. Clin Chim Acta 347: 25-39.

41. Mukhopadhyay R (2004) Don't waste your breath. Researchers are developing breath tests for diagnosing diseases, but how well do they work? Anal Chem 76: 273A-6A.

42. Zlatkis A, Bertsch W, Lichtenstein HA, Tishbee A, Shunbo F, et al. (1973) Profile of volatile metabolites in urine by gas chromatography-mass spectrometry. Anal Chem 45: 763-7.

43. Walsh J (2004) Spectroscopy (Amsterdam) 19: 50

44. He S, Liu J, Liu M (2013) The SAW gas chromatograph and its applications in the public security. Proceedings of IEEE International Conference on Green Computing and Communications and IEEE Internet of Things and IEEE Cyber, Physical and Social Computing. 
45. Manginell RP, Pimentel AS, Mowry CD, Mangan MA, Moorman MW, et al. (2013) Diagnostic potential of the pulsed discharged helium ionization detector (PDHID) for pathogenic Mycobacterial volatile biomarkers. J Breath Res 7: 037107.

46. Jha SK, Yadava RDS (2011) Statistical pattern analysis assisted selection of polymers for odor sensor array. Proceedings of Signal Processing, Communication, Computing and Networking Technologies.

47. Wongchoosuk C, Lutz M, Kerdcharoen T (2009) Detection and classification of human body odor using an electronic nose. Sensors 9: 7234-49.

48. Johnson ATC, Khamis SM, Preti G, Kwak J, Gelperin A (2010) DNA-coated nanosensors for breath analysis. IEEE Sensors Journal 10: 159-66.

49. Liu C, Furusawa Y, Hayashi K (2013) Development of a fluorescent imaging sensor for the detection of human body sweat odor. Sensors and Actuators B: Chemical 183: 117-23.

50. Pearce TC (1997) Computational parallels between the biological olfactory pathway and its analogue the electronic nose': Part II. Sensor-based machine olfaction. Biosystems 41: 69-90.

51. Gopel W (1998) Chemical imaging: I. Concepts and visions for electronic and bioelectronic noses. Sensors and Actuators B: Chemical 52: 125-42.

52. Gardner JW, Bartlett PN (1994) A brief history of electronic noses. Sensors and Actuators B: Chemical 18: 210-1.

53. Pearce TC, Schiffman SS, Nagle HT, Gardner JW (2003) Handbook of Machine Olfaction: Electronic Nose Technology. Wiley-VCH, Weinheim, Germany.

54. Westhoff M, Litterst P, Freitag L, Baumbach JI (2007) Ion mobility spectrometry in the diagnosis of sarcoidosis: results of a feasibility study. J Physiol Pharmacol 58: 739-51.

55. Borsdorf H, Rämmler A (2005) Continuous on-line determination of methyl tert-butyl ether in water samples using ion mobility spectrometry. J Chromatogr A 1072: 45-54.

56. Ruzsanyi V, Sielemann S, Baumbach JI (2007) Detection of sulfur-free odorants in natural gas using ion mobility spectrometry. J Environ Monit 9: 61-5.

57. Ruzsanyi V, Baumbach JI, Sielemann S, Litterst P, Westhoff M, et al. (2005) Detection of human metabolites using multi-capillary columns coupled to ion mobility spectrometers. J Chromatogr A 1084: 145-51.

58. Westhoff M, Freitag PLL, Ruzsanyi V, Bader S, Urfer W, et al. (2005) Ion Mobility Spectrometry: A new method for the detection of lung cancer and airway infection in exhaled air? First results of a pilot study. Chest 128:155S.

59. Perl T, Carstens E, Hirn A, Quintel M, Vautz W, et al. (2009) Determination of serum propofol concentrations by breath analysis using ion mobility spectrometry. Br J Anaesth 103: 822-7.

60. Baumbach JI (2006) Process analysis using ion mobility spectrometry. Anal Bioanal Chem 384: 1059-70.

61. Ruzsanyi V, Baumbach JI (2005) Analysis of human breath using IMS. Int J Ion Mobil Spectrom 8: 5-7.

62. Bödeker B, Vautz W, Baumbach JI (2008) Peak finding and referencing in MCC/IMS-Data. Int J Ion Mobil Spectrom 11: 83-7.

63. Macias MS, Guerra-Diaz P, Almirall JR, Furton KG (2010) Detection of piperonal emitted from polymer controlled odor mimic permeation systems utilizing Canis familiaris and solid phase microextraction-ion mobility spectrometry. Forensic Sci Int 195: 132-8.

64. Xie Z, Ruzsanyi V, Sieleman S, Schmidt H, Baumbach JI (2001) Determination of pentane, isoprene and acetone using HSCC-UV-IMS. Int J Ion Mobil Spectrom 2: 88-91.

65. Huo R, Agapiou A, Bocos-Bintintan V, Brown LJ, Burns C, et al. (2011) The trapped human experiment. J Breath Res 5: 046006.

66. Oh SY (2013) Fast gas chromatography-surface acoustic wave sensor: An effective tool for discrimination and quality control of Lavandula species. Sensors and Actuators B: Chemical 182: 223-31.

67. Itoh T, Wang J, Matsubara I, Shin W, Izu N, et al.(2008) VOCs sensing properties of layered organic-inorganic hybrid thin films: $\mathrm{MoO}_{3}$ with various interlayer organic components. Materials Letters 62: 3021-3.

68. Staples EJ (2000) Real time characterization of food \& beverages using an electronic nose with 500 orthogonal sensors and VaporPrintTM imaging. Sensors Exposition Conference.

69. Sandia National Laboratories (2013) Researching new detectors for chemical, biological threats. Science Daily: 6 September 2013.

70. Wilson AD, Baietto M (2009) Applications and advances in electronic-nose technologies. Sensors 9: 5099-148.

71. Chen X, Cao M, Li Y, Hu W, Wang P, et al. (2005) A study of an electronic nose for detection of lung cancer based on a virtual SAW gas sensors array and imaging recognition method. Meas Sci Technol 16: 1535-46.

72. Dorozhkin LM, Rozanov IA (2001) Acoustic wave chemical sensors for gases. Analytical Chemistry 56: 399-416.

73. Shafiqul Islam AKM, Ismail Z, Ahmad MN, Saad B, Othman AR, et al. (2005) Transient parameters of a coated quartz crystal microbalance sensor for the detection of volatile organic compounds (VOCs). Sensors and Actuators B 109: 238-43.

74. Mensitieri G, Venditto V, Guerra G (2003) Polymeric sensing films absorbing organic guests into a nanoporous host crystalline phase. Sensors and Actuators B 92: 255-61.

75. Penza M, Cassano G, Aversa P, Antolini F, Cusano A, et al. (2005) Carbon nanotubes-coated multi-transducing sensors for VOCs detection. Sensors and Actuators B 111-112: 171-80.

76. Matsuguchi M, Uno T (2006) Molecular imprinting strategy for solvent molecules and its application for QCM-based VOC vapor sensing. Sensors and Actuators B 113: 94-9.

77. Hodgson AT, Levin H (2003) Volatile organic compounds in indoor air: A review of concentrations measured in North America since 1990. Lawrence Berkeley National Laboratory: 1-31.

78. Wolkoff P, Nielsen GD (2001) Organic compounds in indoor air-their relevance for perceived indoor air quality? Atmospheric Environment 35: 4407-17.

79. Haeringer D, Goschnick J (2008) Characterization of smelling contaminations on textiles using a gradient microarray as an electronic nose. Sensors and Actuators B 132: 644-9.

80. Chapman EA, Thomas PS, Stone E, Lewis C, Yates DH (2012) A breath test for malignant mesothelioma using an electronic nose. Eur Respir J $40: 448-54$. 81. Wilson AD, Oberle CS, Oberle DF (2013) Detection of off-flavor in catfish using a conducting polymer Electronic-Nose technology. Sensor 13: 15968-84 
82. Fam DWM, Palaniappan Al, Tok AIY, Liedberg B, Moochhala SM (2011) A review on technological aspects influencing commercialization of carbon nanotube sensors. Sensors and Actuators B: Chemical 157: 1-7.

83. Haddad R, Medhanie A, Roth Y, Harel D, Sobel N (2010) Predicting odor pleasantness with an electronic nose. PLos Comput Biol 6: e1000740.

84. Riffell JA, Abrell L, Hildebrand JG (2008) Physical processes and real-time chemical measurement of the insect olfactory environment. J Chem Ecol 34: 83753.

85. Christian TJ, Kleiss B, Yokelson RJ, Holzinger R, Crutzen PJ, et al (2004) Comprehensive laboratory measurements of biomass-burning emissions: 2. First intercomparison of open-path FTIR, PTR-MS, and GC-MS//FID//ECD. J Geophys Res 109: 1-12.

86. Cias P, Wang C, Dibble TS (2007) Absorption cross-sections of the C-H overtone of volatile organic compounds: 2 methyl-1,3-butadiene (isoprene),

1,3-butadiene, and 2,3-dimethyl-1,3-butadiene. Appl Spectrosc 61: 230-6.

87. Cullen P, Edelman GJ, Van Leeuwen TG, Aalders MC, Gaston E (2012) Hyperspectral imaging for non-contact analysis of forensic traces. Forensic Science International 22nd. October, 2012.

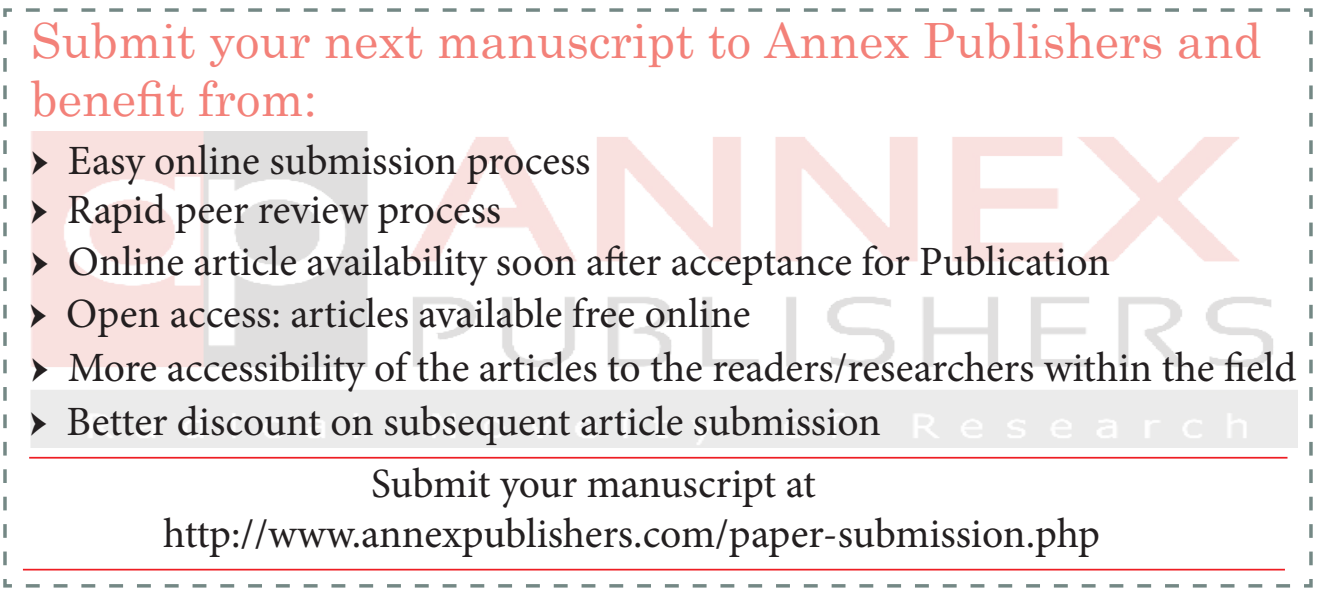

